\title{
Correction to: A Statistical Study of Platelet Size Decomposition and Related Growth Model
}

\author{
Ratan Dasgupta, Koushik Biswas and Debabrata Dash
}

\section{Correction to: \\ Chapter "A Statistical Study of Platelet Size Decomposition and Related Growth Model" in: R. Dasgupta (ed.), Advances in Growth Curve and Structural Equation Modeling, https://doi.org/10.1007/978-981-13-0980-9_11}

The original version of the chapter was inadvertently published without authors' names and their affiliations, Dr. Koushik Biswas, Regional Institute of Ophthalmology, Medical College Hospital, Kolkata-700073, India. Email: koushik2907@gmail.com and Dr. Debabrata Dash, Department of Biochemistry, Institute of Medical Sciences, Banaras Hindu University, Varanasi 221005, India. Email: ddash.biochem@gmail.com in the opening page of chapter "A Statistical Study of Platelet Size Decomposition and Related Growth Model", which have now been included. The correction chapter and the book have been updated with the changes. 